\title{
gु
}

\section{Luttinger Liquid of Photons and Spin-Charge Separation in Hollow-Core Fibers}

\author{
Dimitris G. Angelakis, ${ }^{1,2, *}$ Mingxia Huo, ${ }^{2}$ Elica Kyoseva, ${ }^{2}$ and Leong Chuan Kwek ${ }^{2,3}$ \\ ${ }^{1}$ Science Department, Technical University of Crete, Chania, Crete, Greece, 73100 \\ ${ }^{2}$ Centre for Quantum Technologies, National University of Singapore, 2 Science Drive 3, Singapore 117542 \\ ${ }^{3}$ National Institute of Education and Institute of Advanced Studies, Nanyang Technological University, 1 Nanyang Walk, \\ Singapore 637616 \\ (Received 23 July 2010; revised manuscript received 9 February 2011; published 11 April 2011)
}

\begin{abstract}
In this work we show that light-matter excitations (polaritons) generated inside a hollow-core onedimensional fiber filled with two types of atoms, can exhibit Luttinger liquid behavior. We first explain how to prepare and drive this quantum-optical system to a strongly interacting regime, described by a bosonic two-component Lieb-Liniger model. Utilizing the connection between strongly interacting bosonic and fermionic systems, we then show how spin-charge separation could be observed by probing the correlations in the polaritons. This is performed by first mapping the polaritons to propagating photon pulses and then measuring the effective photonic spin and charge densities and velocities by analyzing the correlations in the emitted photon spectrum. The necessary regime of interactions is achievable with current quantum-optical technology.
\end{abstract}

DOI: 10.1103/PhysRevLett.106.153601

PACS numbers: $42.50 . G y, 71.10 . P m, 71.36 .+c$

Introduction.-One of the most counterintuitive characteristics of one-dimensional electron gases is spin-charge separation. In this case the electrons cease to behave as single particles, and collective excitations appear carrying only charge (and no spin) or only spin (and no charge), which propagate through the system with different velocities [1]. Efforts to observe spin-charge separation include among others the seminal tunneling experiments in metallic chains, organic conductors and carbon nanotubes [2], and more recently in one-dimensional copper oxide systems and quantum wires [3].

In parallel with the studies of strongly correlated phenomena in real condensed matter systems, works in artificially engineered many-body systems have emerged in the last two decades. In the latter, many-body effects could be reproduced and simulated in well controllable environments. Cold atoms and ion traps are the most famous example [4]. Proposals to observe spin-charge separation have also been in place in cold atoms, including both bosonic and fermionic species [5]. However, the lack of necessary individual accessibility and measurement, and the challenges in trapping and cooling especially fermionic gases make current results unclear so far.

Strongly interacting photons (SIPs), as hybrid lightmatter quantum simulators, promise to provide the necessary extra manipulation and measurement that are so far lacking from other proposals. Motivated by significant advances in both the fields of cavity QED and quantum nonlinear optics [6,7], arrays of coupled resonators were proposed initially for photonic gates [8] and then for the quantum simulation of photonic and polaritonic Mott transitions [9]. More recently among other works, the crystallization of photons has also been proposed in a nonlinear fiber [10] and studies of the properties of driven dissipative arrays have appeared [11]. We show here that spin-charge separation could be efficiently observed in a hollow fiber filled with atoms where stationary light-matter polaritons [7] could behave as a quantum Luttinger liquid. We first describe how to prepare and drive the system to a regime where a two bosonic component Lieb-Liniger model is generated. Utilizing the connection between strongly interacting bosonic and fermionic systems [5], we proceed by showing how to identify and measure the effective photonic spin and charge densities and velocities through standard optical methods.

System.-Consider a waveguide, a hollow-core fiber to be more specific [12], filled with two types of atomic gases $a$ and $b$ (two isotopes of $\mathrm{Rb}$ atoms, for example). Two quantum light fields and two classical fields $\hat{E}_{1, \pm}(z, t)$, $\hat{E}_{2, \pm}(z, t)$ and $\Omega_{1, \pm}(t), \Omega_{2, \pm}(t)$ respectively can propagate towards the left and right directions and couple to the atoms as shown in Fig. 1. Initially two resonant optical pulses carried by $\hat{E}_{1,+}(z, t), \hat{E}_{2,+}(z, t)$ are incident from one direction, say the left side. They are injected into the waveguide with the copropagating control fields $\Omega_{1,+}(t)$ and $\Omega_{2,+}(t)$ initially turned on. Hereafter, we will omit the explicit time and spatial dependence of the field operators for economy of notations. The Hamiltonian in the Schrödinger picture can be expressed as a sum of two independent parts describing the evolution of the different atomic species $H=H^{a}+H^{b}$ with

$$
\begin{aligned}
H^{x}= & -\hbar n_{z}^{x} \sum_{i=1}^{2} \int d z\left\{-\omega_{33}^{x} \sigma_{33}^{x}+\left(-\omega_{\mathrm{qu}}^{(i)}+\Delta_{2}^{x}\right) \sigma_{22}^{x}\right. \\
& +\left(-\omega_{33}^{x}-\omega_{\mathrm{qu}}^{(i)}-\Delta_{4}^{x}\right) \sigma_{44}^{x}+g_{i}^{x} \sqrt{2 \pi}\left(\sigma_{21}^{x}+\sigma_{43}^{x}\right) \\
& \times\left(\hat{E}_{i,+} e^{i\left(k_{\mathrm{qu} z}^{(i)}-\omega_{\mathrm{qu}}^{(i)} t\right)}+\hat{E}_{i,-} e^{i\left(-k_{\mathrm{qu}}^{(i)} z-\omega_{\mathrm{qu}}^{(i)} t\right)}\right) \\
& \left.+\left(\Omega_{i,+} e^{i\left(k_{\mathrm{cl}}^{(i)} z-\omega_{\mathrm{cl}}^{(i)} t\right)}+\Omega_{i,-} e^{-i\left(k_{\mathrm{cl}}^{(i)} z+\omega_{\mathrm{cl}}^{(i)} t\right)}\right) \sigma_{23}^{x}+\text { H.c. }\right\}
\end{aligned}
$$




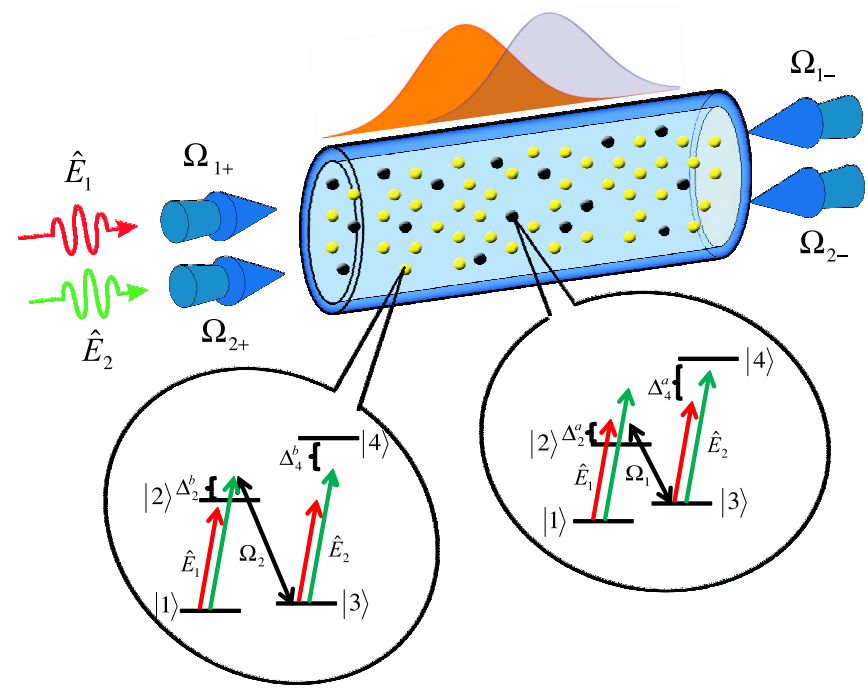

FIG. 1 (color online). A schematic diagram of the system under consideration. A hollow fiber with two quantum light fields $\hat{E}_{1,+}, \hat{E}_{2,+}$ and two pairs of classical fields $\Omega_{1, \pm}$ and $\Omega_{2, \pm}$ propagating towards the left and right directions. The fiber is filled with two atomic gases $a$ and $b$. Appropriate tuning of the couplings of the light fields to the corresponding atomic transitions, forces the trapped polaritons to behave as an effective 1D quantum Luttinger liquid. The available tunability of the effective interaction parameters allows us to reach the spin-charge separation regime. Coherently transferring the polaritons' correlations to propagating light pulses and allowing them to exit the fiber, provides for the efficient measurement of both the dynamics of the propagation of the effective spin and charge quasiparticles or of the spectral function characteristic of the effect taking place.

where $x=\{a, b\}$ labels the two atomic species $a$ and $b$. Here, the continuous collective atomic spin operators $\sigma_{p q}^{x}=\sigma_{p q}^{x}(z, t)(p, q=\{1, \ldots, 4\})$ describe the average of $|p\rangle^{x}\left\langle\left. q\right|^{x}\right.$ over the atoms of type $x$ in a small but macroscopic region around spatial coordinate $z$ and $g_{i}^{x}$ are the coupling between the atomic spin operators and the quantum fields. The densities of the two species of atoms in the same region are assumed to be different and equal to $n_{z}^{a}$ and $n_{z}^{b}$ respectively.

Moreover we use $i=\{1,2\}$ to label the two quantum and two classical fields with frequencies $\omega_{\mathrm{qu}}^{(i)}$ and $\omega_{\mathrm{cl}}^{(i)}$ and wave vectors $k_{\mathrm{qu}}^{(i)}$ and $k_{\mathrm{cl}}^{(i)}$, respectively. Both quantum fields $\hat{E}_{1, \pm}$ and $\hat{E}_{2, \pm}$ drive four possible atomic transitions and for simplicity we assume that each field couples with the same strength to the two transitions of the different atoms. The field $\hat{E}_{1, \pm}(z, t)$ is detuned by $\Delta_{2}^{a}$ from the transition $|1\rangle^{a} \rightarrow|2\rangle^{a}$ and by $\Delta_{4}^{a}$ from $|3\rangle^{a} \rightarrow|4\rangle^{a}$. Similarly, the quantum field $\hat{E}_{2, \pm}(z, t)$ is off-resonant from $|1\rangle^{b} \rightarrow|2\rangle^{b}$ and $|3\rangle^{b} \rightarrow|4\rangle^{b}$ by $\Delta_{2}^{b}$ and $\Delta_{4}^{b}$ as shown on Fig. 1. Finally, the applied classical control beams with Rabi frequencies $\Omega_{1, \pm}(t)$ and $\Omega_{2, \pm}(t)$ couple to both atoms and drive the transitions $|3\rangle^{x} \rightarrow|2\rangle^{x}$.

The evolutions of the slowly varying quantum operators $\hat{E}_{i, \pm}(z, t)$ are given by four Maxwell-Bloch (MB) equa- tions: $\left(\partial_{t} \pm \nu^{(1)} \partial_{z}\right) \hat{E}_{1, \pm}=i \sqrt{2 \pi} n_{z}^{a} g_{1}^{a}\left(\sigma_{12, \pm}^{a}+\sigma_{34, \pm}^{a}\right)$ and $\left(\partial_{t} \pm \nu^{(2)} \partial_{z}\right) \hat{E}_{2, \pm}=i \sqrt{2 \pi} n_{z}^{b} g_{2}^{b}\left(\sigma_{12, \pm}^{b}+\sigma_{34, \pm}^{b}\right)$ where we have introduced the slowly varying collective operators $\sigma_{p q}^{a}=\sigma_{p q,+}^{a}(z, t) e^{i k_{\mathrm{qu}}^{(1)} z}+\sigma_{p q,-}^{a}(z, t) e^{-i k_{\mathrm{qu}}^{(1)} z} \quad$ and $\quad \sigma_{p q}^{b}=$ $\sigma_{p q,+}^{b}(z, t) e^{i k_{\mathrm{qu}}^{(2)} z}+\sigma_{p q,-}^{b}(z, t) e^{-i k_{\mathrm{qu}}^{(2)} z}$. Here, $\nu^{(1,2)}$ are the velocities for each quantum field in an empty waveguide. In the derivation of the equations of motion we have assumed the Rabi frequencies of the control fields to be slowly varied and ignored the high-frequency terms oscillating at frequency $2 \Delta_{2}^{x} t$ following the methods in [7]. We proceed to define the polariton operators as $\Psi_{1, \pm}=$ $\cos \theta^{a} \hat{E}_{1, \pm}-\sin \theta^{a} \sqrt{2 \pi n_{z}^{a}} \sigma_{31}^{a}$ and $\Psi_{2, \pm}=\cos \theta^{b} \hat{E}_{2, \pm}-$ $\sin \theta^{b} \sqrt{2 \pi n_{z}^{b}} \sigma_{31}^{b} \quad$ where $\tan \theta^{a}=g_{1}^{a} \sqrt{2 \pi n_{z}^{a}} / \Omega_{1} \quad$ and $\tan \theta^{b}=g_{2}^{b} \sqrt{2 \pi n_{z}^{b}} / \Omega_{2}$. For simplicity we have assumed that the amplitudes of the counterpropagating classical fields are equal, i.e., $\Omega_{i, \pm} \equiv \Omega_{i}(i=1,2)$. In the limit when the excitations are mostly in spin-wave form, i.e., $\sin \theta^{x} \simeq 1$ and since $\sigma_{31}^{a}=-g_{1}^{a} \hat{E}_{1, \pm} / \Omega_{1}$ and $\sigma_{31}^{b}=$ $-g_{2}^{b} \hat{E}_{2, \pm} / \Omega_{2}$, the polariton operators are $\Psi_{1, \pm}=$ $g_{1}^{a} \sqrt{2 \pi n_{z}^{a}} \hat{E}_{1, \pm} / \Omega_{1}$ and $\Psi_{2, \pm}=g_{2}^{b} \sqrt{2 \pi n_{z}^{b}} \hat{E}_{2, \pm} / \Omega_{2}$.

Setting $\Psi_{1,2}=\left(\Psi_{1,2 ;+}+\Psi_{1,2 ;-}\right) / 2$ and $A_{1,2}=$ $\left(\Psi_{1,2 ;+}-\Psi_{1,2 ;-}\right) / 2$ as the symmetric and antisymmetric combinations of the two polaritons and using the $\mathrm{MB}$ equations from earlier, we derive the equations of motion for the polariton combination $\Psi_{1}, A_{1}$ :

$$
\begin{aligned}
\partial_{t} \Psi_{1}+\nu^{(1)} \partial_{z} A_{1}= & -\sqrt{2 \pi} \frac{\left(g_{1}^{a}\right)^{2}}{2 \Omega_{1}^{2}} n_{z}^{a} \partial_{t} \Psi_{1} \\
& -i \frac{\left(g_{1}^{a}\right)^{2}}{\sqrt{2 \pi} \Delta_{4}^{a}}\left(2 \Psi_{1}^{\dagger} \Psi_{1}+A_{1}^{\dagger} A_{1}\right) \Psi_{1} \\
& -i \frac{\left(g_{1}^{a}\right)^{2}\left(g_{2}^{a}\right)^{2} \Omega_{2}^{2}}{\sqrt{2 \pi}\left(g_{1}^{b}\right)^{2} \Delta_{4}^{a} \Omega_{1}^{2}} \\
& \times\left(\Psi_{2}^{\dagger} \Psi_{2}+A_{2}^{\dagger} A_{2}\right) \Psi_{1}+\text { noise, } \\
\partial_{t} A_{1}+\nu^{(1)} \partial_{z} \Psi_{1}= & -i \sqrt{2 \pi} \frac{\left(g_{1}^{a}\right)^{2}}{\Delta_{2}^{a}} n_{z}^{a} A_{1}-\frac{\left(g_{1}^{a}\right)^{2}}{\sqrt{2 \pi} \Delta_{4}^{a}} \Psi_{1}^{\dagger} \Psi_{1} A_{1} \\
& + \text { noise. }
\end{aligned}
$$

A similar set of equations hold for the pair $\Psi_{2}, A_{2}$. The noise terms in Eqs. (2) account for the dissipative processes that take place during the evolution. Fortunately, for the dark state polaritons under consideration, as long as the spontaneous emission rates $\Gamma$ from the states $|2\rangle$ and $|4\rangle$ are much less than the detuning $\left|\Delta_{4}^{a}\right|,\left|\Delta_{4}^{b}\right|$, the losses in the time scales of interest are not significant and thus can be neglected $[7,10]$. Assuming sufficient optical depth of a few thousand and a large ratio between the density of atoms to the density of photons $n_{z}^{i} / \rho_{0, i} \sim 10^{4}$, the antisymmetric combinations $A_{1}$ and $A_{2}$ can be adiabatically eliminated from the equations of motion for the polaritons and moreover, the nonlinear terms $\Psi_{1}^{\dagger} \Psi_{1} A_{1}, \Psi_{2}^{\dagger} \Psi_{2} A_{2}$ are 
negligible. Then, Eqs. (2) simplify to $i \partial_{t} \Psi_{1}=$ $-\frac{1}{2 m_{1}} \partial_{z}^{2} \Psi_{1}+U_{1} \Psi_{1}^{\dagger} \Psi_{1} \Psi_{1}+V_{1} \Psi_{2}^{\dagger} \Psi_{2} \Psi_{1}$ and $i \partial_{t} \Psi_{2}=$ $-\frac{1}{2 m_{2}} \partial_{z}^{2} \Psi_{2}+U_{2} \Psi_{2}^{\dagger} \Psi_{2} \Psi_{2}+V_{2} \Psi_{1}^{\dagger} \Psi_{1} \Psi_{2}$. These are the equations of motion of a two-component Lieb-Liniger model (LL) of polaritons with the corresponding Hamiltonian:

$$
\begin{aligned}
H= & \hbar \int d z\left\{\sum_{i}\left[\frac{1}{2 m_{i}} \partial_{z} \Psi_{i}^{\dagger}(z) \partial_{z} \Psi_{i}(z)+\frac{U_{i}}{2} \rho_{i}^{2}(z)\right]\right. \\
& \left.+V_{12} \rho_{1}(z) \rho_{2}(z)\right\} .
\end{aligned}
$$

$m_{i}$ is effective mass for the $i$ th polariton with $\frac{1}{m_{1}}=-\frac{4 \Delta_{2}^{a} v_{z}^{(1)}}{\Gamma_{1 \mathrm{D}}^{a} n_{z}^{a}}$ and $\frac{1}{m_{2}}=-\frac{4 \Delta_{2}^{b} \nu_{g}^{(2)}}{\Gamma_{1 \mathrm{D}}^{b} n_{z}^{b}}$ with $\Gamma_{1 \mathrm{D}}^{a, b}$ the spontaneous emission rate of a single atom in the waveguide modes. $\nu_{g}^{(1)}=$ $\nu^{(1)} \Omega_{1}^{2} / \pi\left(g_{1}^{a}\right)^{2} n_{z}^{a}$ and $\nu_{g}^{(2)}=\nu^{(2)} \Omega_{2}^{2} / \pi\left(g_{2}^{b}\right)^{2} n_{z}^{b}$ are the corresponding group velocities of the propagating polaritons. The intraspecies repulsions are given by $U_{1}=\frac{\Gamma_{1 \mathrm{D}} \nu_{g}^{(1)}}{2 \Delta_{4}^{a}}$, $U_{2}=\frac{\Gamma_{1 \mathrm{D}} \nu_{g}^{(2)}}{2 \Delta_{4}^{b}}$ and the interspecies repulsions by $V_{12}=$ $\frac{\pi\left(g_{1}^{a}\right)^{2}\left(g_{2}^{a}\right)^{2} \nu_{g}^{(1)}}{\left(g_{2}^{b}\right)^{2} \Delta_{4}^{a} \nu^{(1)}}+\frac{\pi\left(g_{2}^{b}\right)^{2}\left(g_{1}^{b}\right)^{2} \nu_{g}^{(2)}}{\left(g_{1}^{a}\right)^{2} \Delta_{4}^{b} \nu^{(2)}}$.

Let us now define the effective parameters $u_{i}=$ $\sqrt{\rho_{0, i} U_{i} / m_{i}}$ and $K_{i}=\sqrt{\pi^{2} \rho_{0, i} /\left(m_{i} U_{i}\right)}$ with $\rho_{0, i}$ equal to $n_{\mathrm{ph}, i}$ the photon number of $i$ th quantum field in our scheme. It is known from the works by Girardeau and others [1], that when $u_{1}=u_{2}=u$ and $K_{1}=K_{2}=K$, the above LL Hamiltonian Eq. (3) can be transformed into a Luttinger liquid one comprising separately of two parts, the charge part $H_{c}$ and the spin part $H_{s}$ as $H=H_{c}+H_{s}$. The latter allows for the separation of a single excitation into two separate ones each comprising of spins or of charge or density. These can propagate through the liquid with different velocities given by $u_{c, s}=u \sqrt{1 \pm \frac{\left(V_{1}+V_{2}\right) K}{\pi u}}$. In our case, the charge (spin) density corresponds to the sum (difference) of the corresponding polaritons' densities $n_{c, s}=$ $n_{1} \pm n_{2}$ with $n_{i}=\left\langle\Psi_{i}^{\dagger} \Psi_{i}\right\rangle$. Recall that $\Psi_{i}$ is the symmetric combinations of the two counterpropagating dark state polaritons generated by each set of atom-field interaction as defined earlier.

Preparation and detection.-Initially, two probe pulses are sent in from the left and become trapped in the usual slow light manner [6,7]. Labeling $\gamma_{1,2}$ the ratio of the interaction to the kinetic energies for each polariton species, at this stage $\gamma_{1,2}<1$ the pulses are noninteracting with the photons expanding freely due to dispersion. By slowly shifting in the 4th levels, the effective masses $m_{1,2}$ can be kept constant whereas the effective intra- and interspecies repulsions $U_{1,2}$ and $V_{12}$ are increased. This drives the system into a strongly interacting regime with $\gamma_{1,2}>1$ (the bounds on the relevant time scales for the process to be efficient against losses are discussed further down in the text). This dynamic evolution is possible by keeping for example the corresponding two photon detunings $\Delta_{2}^{a}, \Delta_{2}^{b}$ constant, while shifting the level 4 responsible for the nonlinear shifts.

Once this correlated state is achieved, one field-for example $\Omega_{1,+}$-from the pair of control fields that trap polariton type $\Psi_{1}$ is slowly turned off. This will release the corresponding quasiparticle and allow it to propagate towards the exit of the fiber. As all correlations established in the previous step - the evolution under the LL Hamiltonian Eq. (3) - are retained, this wave packet containing mostly light now, comprises of two separated parts propagating with different velocities $u_{s}$ and $u_{c}$ towards the end of the fiber. The detection in our case can occur through dynamically probing the time evolution of a single excitation as in cold atom proposals [5] or by measuring the corresponding single particle spectral function $S(q, \omega)$ as in condensed matter experiments [1-3].

In our case the charge (spin) density waves, after the release of the polaritons, will transfer to the sum (difference) of the corresponding time dependent photon intensities $n_{i}$ for each propagating field where $n_{i}=\left\langle\hat{E}_{i,+}^{\dagger} \hat{E}_{i,+}\right\rangle$. One could dynamically probe the corresponding photonic intensity maxima, and from that infer the corresponding velocities. Alternatively, we could measure the spectral function by probing the first order correlation function of one of the fields, say $\hat{E}_{1}$, for a specific quasimomentum $q$. The latter should exhibit two peaks as shown in the inset of Fig. 2 centered around $u_{s} q$ and $u_{c} q$ with $q$ the corresponding quasimomentum of the propagating excitation. In our case, $q$ is inversely proportional to the initial extend of the pulse $z_{0}$. For clear distinction between the two spin and charge peaks, we should tune our optical detectors around $q=2 \pi / z_{0}$.

In Fig. 2 we plot the spectral function as analytically derived for a two-component bosonic system [13], for intra- and interspecies values corresponding in our system to $u=1$ and $k=1$. Here $U_{1} / V_{12}=U_{2} / V_{12}=0.6$ which in turn give a ratio between the effective charge and spin velocities of $u_{c} / u_{s}=2$. The corresponding necessary values of the parameters in our system are optical depths $\mathrm{OD}=3000$ and roughly $N_{\mathrm{ph}}^{1,2}=10$ photons initially in each pulse and single-atom cooperativity of $\eta=0.4$. We assume the same single-atom cooperativity for both species $\eta^{a, b}=\eta=\Gamma_{1 \mathrm{D}} / \Gamma$, which is the ratio of spontaneous emission into the waveguide to total spontaneous emission.

We note here in calculating the parameters, we take into account that both the linear and the nonlinear loss mechanisms will define a maximum evolution time $t_{\max }$ for the second phase of the process $[7,10]$. This in turn, gives the following condition on the achievable ratio of interaction to kinetic energies: $\gamma_{\max }^{1,2} \sim \min \left[\exp \left(\frac{\left|\Delta_{2}^{a, b}\right|}{\Gamma}, \eta \frac{\Gamma}{\left|\Delta_{2}^{a, b}\right|} \frac{\mathrm{OD}}{N_{\mathrm{ph}}^{a, b}}\right)\right]$. Optimizing over $\Delta_{2}^{a, b}$ will give the numbers mentioned above. We add here that in order to omit the noise terms and also be able to neglect the higher order derivatives so 


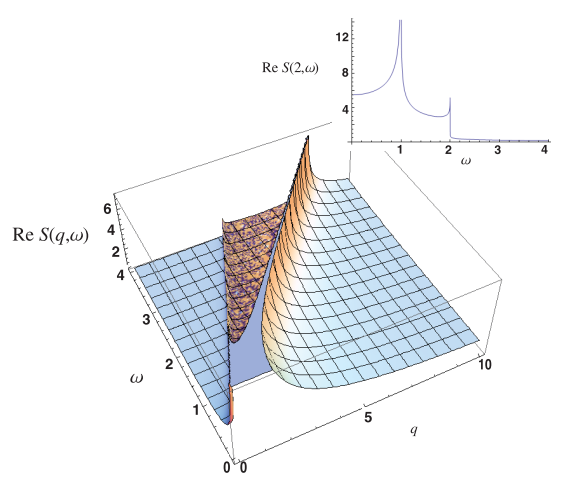

FIG. 2 (color online). The single particle spectral function for our photonic system. The effective spin and charge velocities are $u_{s}=0.5$ and $u_{c}=1$ and can be achieved for optical depths around $\mathrm{OD}=3000$, with 10 photons in each pulse initially, and single-atom cooperativity for each atomic species of 0.4 . Subsequent releasing of one of the trapped polaritons through its coherent mapping to a propagating light pulse, allows for the efficient measurement of the spectral function by measuring cross correlations in the intensities of the output fields. In the inset we plot a 2D cut for the value of $q=2$. The units of $\omega$ and $q$ are in $\frac{\pi}{z_{0}} \times 2 \sqrt{\frac{2}{5}} u$ and $\pi / z_{0}$, respectively (see text).

that the system is described within good approximation by the Lieb-Liniger Eq. (3), the following conditions need to be satisfied: The Stark shift of levels $|2\rangle$ for each atomic species should stay within the transparency window and a bound on spin-wave excitations wave vector should be in place [7]. These conditions translate to $n_{1} / n_{z}^{a} \ll$ $\left|\Delta_{4}^{a}\right| /\left|\Gamma-2 i \Delta_{2}^{a}\right|, \quad n_{2} / n_{z}^{b} \ll\left|\Delta_{4}^{b}\right| /\left|\Gamma-2 i \Delta_{2}^{b}\right| \quad$ and $n_{1} / n_{z}^{a} \ll \Gamma_{1 \mathrm{D}}^{a} /\left|2 \Delta_{2}^{a}+i \Gamma\right|, \quad n_{2} / n_{z}^{b} \ll \Gamma_{1 \mathrm{D}}^{b} /\left|2 \Delta_{2}^{a}+i \Gamma\right|$ which is satisfied in our case as the numbers of atoms can be two or three orders of magnitude larger than the number of photons. We note that in this case the ratio between kinetic and repulsion energies for each species can reach the value of 40 which for the single component case was shown to lead to a Tonks gas of photons [10]. For spin-charge separation, the required repulsions could be of a smaller value, thus relaxing the overall quantum-optical requirements but the two peaks in the spectral function will be less pronounced.

We would like to stress here that our proposal is inherently different to the one proposed in [10] both in terms of the quantum optics scheme under consideration (two atomic species), the measurement part (spectral function accessibility) and also fundamentally. Here we are using photons to simulate a purely fermionic effect, i.e., spincharge separation and not hardcore bosons in one dimension [1]. In our opinion measuring spin-charge separation and directly accessing the spectral function of a Luttinger liquid has proven more cumbersome and has not been clearly experimentally verified yet, in spite seminal efforts in both the condensed matter setups and cold atom simulators [2-4].

In conclusion, we have shown that stationary lightmatter excitations generated inside a hollow one- dimensional waveguide filled with atoms can be made to generate a photonic two-component Lieb-Liniger model simulating a quantum Luttinger liquid. Moreover the relevant interactions exhibit the necessary tunability both to generate and efficiently observe spin-charge separation using standard quantum-optical methods.

We would like to acknowledge financial support by the National Research Foundation \& Ministry of Education, Singapore.

*dimitris.angelakis@gmail.org

[1] M. Girardeau, J. Math. Phys. (N.Y.) 1, 516 (1960); M. Girardeau, Phys. Rev. 139, B500 (1965); T. Giamarchi, Quantum Physics in One Dimension (Oxford University Press, Oxford, 2004); B. Paredes et al., Nature (London) 429, 277 (2004).

[2] C. Kim et al., Phys. Rev. Lett. 77, 4054 (1996); P. Segovia et al., Nature (London) 402, 504 (1999); T. Lorenz et al., Nature (London) 418, 614 (2002).

[3] O. M. Auslaender et al., Science 308, 88 (2005); B. J. Kim et al., Nature Phys. 2, 397 (2006); Y. Jompol et al., Science 325, 597 (2009).

[4] D. Jaksch et al., Phys. Rev. Lett. 81, 3108 (1998); M. Greiner et al., Nature (London) 415, 39 (2002); D. Porras and I. Cirac, Phys. Rev. Lett. 92, 207901 (2004); A. Friedenaue et al., Nature Phys. 4, 757 (2008).

[5] A. Recati et al., Phys. Rev. Lett. 90, 020401 (2003); L. Kecke, H. Grabert, and W. Hausler, Phys. Rev. Lett. 94, 176802 (2005); C. Kollath, U. Schollwöck, and W. Zwerger, Phys. Rev. Lett. 95, 176401 (2005); A. Kleine et al., Phys. Rev. A 77, 013607 (2008).

[6] H. Walther, B. T. H. Varcoe, B.-G. Englert, and T. Becker, Rep. Prog. Phys. 69, 1325 (2006); M. Fleischhauer, A. Imamoglu, and J.P. Marangos, Rev. Mod. Phys. 77, 633 (2005).

[7] M. Fleischhauer and M.D. Lukin, Phys. Rev. Lett. 84, 5094 (2000); M. Bajcsy et al., Nature (London) 426, 638 (2003); M. Bajcsy et al., Phys. Rev. Lett. 102, 203902 (2009).

[8] D. G. Angelakis, M. F. Santos, V. Yannopapas, and A. Ekert, Phys. Lett. A 362, 377 (2007).

[9] M. J. Hartmann, F. G.S. L. Brandão, and M. B. Plenio, Nature Phys. 2, 849 (2006); D. G. Angelakis, M. F. Santos, and S. Bose, Phys. Rev. A 76, 031805(R) (2007); A. D. Greentree et al., Nature Phys. 2, 856 (2006).

[10] D. E. Chang et al., Nature Phys. 4, 884 (2008).

[11] D. Rossini and R. Fazio, Phys. Rev. Lett. 99, 186401 (2007); T. Byrnes et al., Phys. Rev. B 78, 075320 (2008).M. Aichhorn et al., Phys. Rev. Lett. 100, 216401 (2008); Dario Gerace et al., Nature Phys. 5, 281 (2009); I. Carusotto et al., Phys. Rev. Lett. 103, 033601 (2009); D. G. Angelakis et al., Europhys. Lett. 85, 20007 (2009).

[12] S. Ghosh et al., Phys. Rev. Lett. 94, 093902 (2005); K. P. Nayak et al., Opt. Express 15, 5431 (2007); T. Takekoshi and R. J. Knize, Phys. Rev. Lett. 98, 210404 (2007); C. A. Christensen et al., Phys. Rev. A 78, 033429 (2008); S. Vorrath et al., New J. Phys. 12, 123015 (2010).

[13] A. Iucci, G. A. Fiete, and T. Giamarchi, Phys. Rev. B 75, 205116 (2007). 